\title{
Dis, M'sieur, c'est quoi la poésie : Jean-Michel Maulpoix en 10 questions
}

\author{
Jean-Paul Giraux
}

ean-Michel Maulpoix nous propose à travers ses multiples ouvrages et son site Internet, une auvre théorique de première importance. Tous ceux qui l'ont pratiquée ont été séduits par la clarté des démonstrations et l'aptitude de l'auteur à traduire ses idées dans des formules ramassées qui font mouche. On en rencontrera quelques-unes au cours de cet échange.

On verra que ce riche ensemble a pour but essentiel de répondre autant que faire se peut à la question de savoir ce quion peut dire de la poésie et peut-être même ce qu'elle est.

"La poésie, c’est ce que fait le poète " écrivait Machado. Mais encore?

$$
\infty
$$

JPG : $\mathbf{1}^{\circ}$ Avec le temps tout s'en va, c'est bien connu, et le poème ne saurait déroger à cette entropie. Le voilà, nous dit Jean-Michel Maulpoix, " amaigri, appauvri, interdit de Chant, le voici devenu un rude objet de langue moins fait pour émouvoir que pour infuser de l'effroi ". Il ne resterait donc plus qu'à suivre le convoi et poser sur sa tombe la couronne pieusement dédiée au cher disparu. Est-ce bien là le sens de ces " adieux au poème ", titre apocalyptique qui annonce, sinon la fin du monde du moins celle de la poésie?

* Jean-Paul Giraux a pété professeur de lettres dans un collège parisien. Il écrit des poèmes, des nouvelles, des romans. Il anime depuis sa création « Le Mercredi du poète ". et donne régulièrement ses chroniques et ses lectures critiques à des revues de poésie et principalement à Poésie-sur-Seine et Poésie Première. 
JMM : Oui, ces « adieux " expriment une tristesse et une inquiétude : celles de voir s'éteindre peu à peu la poésie, étouffée aussi bien de l'extérieur par l'incessant vacarme des médias que de l'intérieur par son étranglement propre, bien compréhensible celui-là, puisqu'il résulte d'une longue histoire qui a commencé avec les " couacs » rimbaldiens... La poésie n’est pas étrangère aux fluctuations de ce que Mallarmé appelait "l'époque "; en ses ouvres les plus fortes elle donne même à entendre la capacité de la langue à un moment donné de l'histoire : je la vois et l'entends à présent comme une voix sombre, désarticulée, très éloignée du chant, menacée d'extinction...

Toutefois, ces " adieux " manifestent aussi bien une espèce de persévérance, un soin un peu cérémonieux, et, jusque dans l'hommage, le souci de poursuivre malgré tout...

JPG : $2^{\circ} \mathrm{J}-\mathrm{M}$ Maulpoix nous propose, notamment à travers Francis Ponge, Philippe Jaccottet et Michel Deguy ( $c f$. Le poète perplexe), le portrait pittoresque du "poète tardif ", à la fois révolté et exténué, une sorte d'ascenseur qui monte et qui descend en fonction de ses enthousiasmes et de ses dépressions, bref un cyclothymique dont on se demande s'il ne relève pas davantage de la psychiatrie que de la littérature, à moins que l'un et l'autre soient indispensables au bon fonctionnement du poème. Qu'en estil exactement?

JMM : Baudelaire a donné le ton ou le rythme de la modernité dans "l'albatros" : c'est le pas claudiquant! Un chemin en lignes brisées. Le "poète tardif ", tel que Jaccottet par exemple en esquisse la figure, est un poète qui fait effort pour chanter " néanmoins", pour continuer malgré tout ce qui le décourage. Il ne peut s'élancer comme naguère sur les longues rampes fiévreuses du lyrisme : quand il semble prendre un envol, c'est pour rechuter aussitôt, car il est l'observateur critique de son propre chant. D'où cette allure « cyclothymique » qui tient à sa précarité même.

JPG : $3^{\circ}$ Pour évoquer le poète, l'image nullement excentrique mais quand même un peu effrayante de l'araignée est récurrente dans l'ouvre théorique de JM Maulpoix. Comment la justifier de préférence à celles de la taupe ou de l'oiseau qui apparaissent également dans ses propos?

JMM : L'intérêt de l'araignée est évidemment dans son patient travail de tissage, effectué silencieusement, obscurément (" invisiblement » suis-je tenté de dire) dans la patience et l'ennui du temps. Et le tissu auquel 
travaille l'araignée présente au moins trois caractéristiques qui le rapprochent du poème : il est un filet tendu dans l'espace pour attraper au vol des proies, il est un tissu fait de trous (tout comme le poème moderne aménage des vides dans la langue)

JPG : $4^{\circ} \mathrm{J}-\mathrm{M}$ Maulpoix se résout au constat suivant : "Qu'on se le dise : il n'est plus temps d'instruire le procès du lyrisme. La sentence est tombée de longue date : travaux forcés à perpétuité. " (cf. Le poète perplexe). Luimême admettant que le lyrisme est "le mauvais genre de la poésie", comment s'en faire encore le défenseur, y compris sous cette forme "soft » qu'il désigne par "lyrisme critique »?

JMM : J'entends par lyrisme à la fois ce dont la poésie s'alimente et ce dont elle se défend : la force qui la conduit et l'énergie qui lui manque ou l'excès qu'il lui faut combattre... Lyrisme est un beau mot, où se donne à entendre encore le chant perdu de la lyre. C'est même un néologisme qui transfere à même nos voix quotidiennes ("cet homme parle avec lyrisme ») l'énergie poétique. Il fait le pont entre l'écriture et la vie. C'est pourquoi il m'a plu de m’y attacher, à rebours de la doxa de l'époque.

JPG : $5^{\circ}$ Une des caractéristiques de la poésie moderne depuis Baudelaire, nous dit J-M Maulpoix, est d'inscrire "la critique de l'art dans l'art luimême » au point d'en arriver à ce que le poème donne à lire l'histoire de sa fabrication. On sait que $\mathrm{H}$. Meschonnic s'est insurgé d'une manière très polémique contre cette "théâtralisation " du poème qui n'épargne pas ce qu'il appelle les HLM, c'est-à-dire les Habitations à Lyrisme Modéré. Je le cite : "...le vide n'est pas dans les blancs, mais dans ce qui est dit, qui ne dit rien, que son intention de dire... ». Que penser de cette instrumentalisation du poème où le poème semble courir après la poésie comme un joggeur après sa jeunesse, évidemment sans jamais la rattraper?

JMM : Si nostalgique soit-il, le poème ne me paraît pas courir après sa jeunesse. L'âge d'or du chant qu'il évoque (si un tel âge a jamais existé? À la Renaissance? Au début du XIXe siècle?) vaut certes comme une espèce d'étalon or du poème (sa mesure idéale!) mais il représente surtout un point de l'histoire à partir ou en fonction duquel situer l'état présent de la parole poétique...

Je ne crois pas avec Meschonnic que le poème ne dise rien d'autre que son intention de dire : il lui arrive aussi bien de donner à entendre son 
intention de ne pas dire, ou son incapacité à dire, voire de montrer de façon spectaculaire et brutale le silence...

JPG : $6^{\circ}$ Le poète a été longtemps le bras séculier des dieux. Il parlait par sa voix. JM Maulpoix note à ce propos : " Chez Baudelaire se retire et agonise le dieu dont Mallarmé suivra les obsèques ". Doit-on comprendre qu'ayant tordu le cou non pas à l'éloquence mais au verbe divin, il ne reste plus au langage qu'à se sacraliser à travers la poésie? Serait-on passé - on me pardonnera ce vilain jeu de mots - du poème à Dieu aux adieux au poème?

JMM : Ce jeu de mots a du sens, même si je ne crois guère à quelque sacralisation de la poésie, ni du langage. La dimension humaine (et non divine) est notre lot. Nous avons encore beaucoup à apprendre, de ce côté, pour la rejoindre.

JPG : $7^{\circ} \mathrm{J}-\mathrm{M}$ Maulpoix observe que le poète découpe la page, la met en scène, s'opposant en cela au romancier qui joue sur le continu, les effets de masse. Il a cette belle formule qu'on va lui demander de commenter : « $L \boldsymbol{e}$ poète est celui qui cherche à en "découdre" ".

JMM : Certains champs lexicaux sont riches de ressources pour parler de la poésie. Ainsi celui du lien, de la corde, du tissage, de la toile d'araignée, etc. Couper-lier sont deux maitres mots du travail poétique. Coudre en découdre en sont deux autres : avec ce dernier verbe, c'est l'idée de crise, d'affrontement qui apparaît. Il y a tension, conflit entre désir et principe de réalité, opposition de « loyaux adversaires »...

JPG : $8^{\circ}$ Une question est posée : la poésie s'est-elle constituée en «idiome séparé ", un peu comme la philosophie avec laquelle elle entretient des rapports de plus en plus étroits. Pourtant remarque J-M Maulpoix, s'il faut au philosophe des idées, au poète, il faut " des mots qui se prennent pour des choses». Et il a cette formule heureuse : "Le philosophe ne veut pas être dupe de ce qui se joue à son insu dans sa conscience, le poète ne peut qu'accepter cet inconnu et lui obéir ». Commentaires?

JMM : Là réside pour une part, il me semble, la pomme de discorde entre poésie et philosophie, au moins dans la conception platonicienne... Le philosophe accepte mal un art qui pour une part se fonde sur les affects, les 
crises, les pulsions, un art dont la richesse tient à la quantité de sombres matériaux qu’il charrie.

JPG : $9^{\circ}$ Jean-Michel Maulpoix accorde une attention soutenue au mythe d'Orphée dans lequel il voit une personnification forte du poète, pas tellement à cause de la lyre et du chant, mais en raison de son irrépressible "retournement » qui fait de lui un spécialiste du ratage (sa vie, ses amours), un préposé à la nostalgie (mes amis, ma jeunesse, le temps perdu) et au désespoir (il est toujours en deuil de quelque chose observe J-M Maulpoix). $\mathrm{Si}$ on ajoute son attachement à la langue, le poète ne serait-il pas quelque chose comme un réactionnaire de nature, au moins un conservateur? Et l'avenir dans tout ça

JMM : À la suite d'Orphée, certes le poète se retourne beaucoup (" Je me retournerai souvent " s'exclame Apollinaire), mais il est aussi celui qui « va plus avant ", celui qui chante comme Rimbaud ou Char l'en allée matinale et la vigueur future. La poésie en appelle à la vie : elle la presse d'advenir et de se déclarer. Et si elle regarde en arrière, ce n'est pas seulement pour déplorer un âge plus jeune et vigoureux : c'est aussi pour y retrouver des raisons de croire en l'avenir, voire la force de s'élancer vers lui...

JPG : $10^{\circ}$ On observe que les trois gros essais publiés chez Corti entre 2002 et 2005 - Du lyrisme - Le poète perplexe et Adieux au poème appartiennent à une collection sous-titrée "en lisant et en écrivant 》. Faudrait-il en déduire que l'œuvre théorique de Jean-Michel Maulpoix son œuvre critique - est avant tout un éloge de la lecture et singulièrement de la lecture du poème?

JMM : Oui, sans doute. De même que l'on a pu montrer que la figure du " traducteur " était centrale chez Jaccottet (qui a fait de la traduction son métier tout comme il s'est posé modestement en traducteur de ses propres promenades), je crois la figure du lecteur essentielle à la définition du poète qui est bien plus que "le propre lecteur de lui-même ". 